Research Paper

\title{
The clinical impact of Hangeshashinto (TJ-14) in the treatment of chemotherapy-induced oral mucositis in gastric cancer and colorectal cancer: Analyses of pooled data from two phase II randomized clinical trials (HANGESHA-G and HANGESHA-C)
}

\author{
Kazuhiro Nishikawa1 ${ }^{*}$, Toru Aoyama ${ }^{2 \llbracket *}$, Mari S Oba ${ }^{3}$, Takaki Yoshikawa2, Chu Matsuda4, Yoshinori \\ Munemoto ${ }^{5}$, Nobuhiro Takiguchi ${ }^{6}$, Kazuaki Tanabe$^{7}, \mathrm{Naoki}_{\mathrm{Nagata}}^{8}$, Motohiro Imano ${ }^{9}$, Mitsuru Oshiro ${ }^{10}$, \\ Ryoji Fukushima11, Masato Kataoka ${ }^{12}$, Satoshi Morita ${ }^{13}$, Akira Tsuburaya14, Hideyuki Mishima15, Toru \\ Kono $^{16}$, Junichi Sakamoto ${ }^{17}$
}

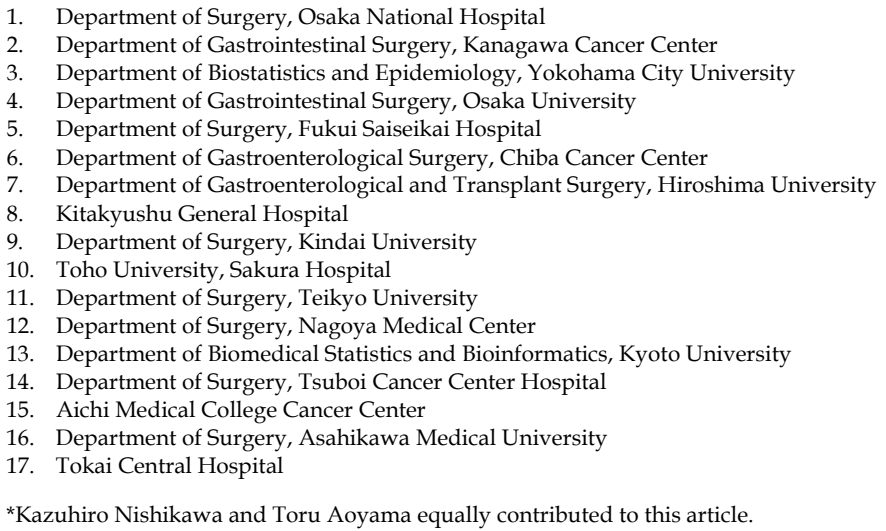

$\triangle$ Corresponding author: Toru Aoyama, Department of Gastrointestinal Surgery, Kanagawa Cancer Center

(C) Ivyspring International Publisher. This is an open access article distributed under the terms of the Creative Commons Attribution (CC BY-NC) license (https://creativecommons.org/licenses/by-nc/4.0/). See http://ivyspring.com/terms for full terms and conditions.

Received: 2018.01.03; Accepted: 2018.03.04; Published: 2018.04.19

\begin{abstract}
Background: The current pooled analysis evaluated the efficacy of Hangeshashinto (TJ-14) in the prevention and/or treatment of chemotherapy-induced oral mucositis (COM) in gastric cancer and colorectal cancer using two prospective, multi-institutional, randomized, double-blind, placebo-controlled phase II trials.

Patients and Methods: HANGESHA-G and HANGESHA-C randomly assigned patients with gastric cancer or colorectal cancer who developed moderate to severe COM (grade $\geq 1$ ) during any cycle of chemotherapy to receive either TJ-14 or a placebo as a double-blind trial. The patients received a placebo or TJ-14 for four to six weeks, according to the chemotherapy regimen, from the start of their next course of chemotherapy. The primary endpoint was the incidence of grade $\geq 2$ COM in the protocol treatment course, and the secondary endpoints were the time to disappearance of COM and the incidence of adverse events.

Results: The pooled population included 181 patients. The incidence of grade $\geq 2$ COM in the TJ-14 group was $55.7 \%$ ( 49 patients), while that in the placebo group was $53.8 \%$ ( 50 patients); there was no significant difference between the two groups $(p=0.796)$. The median time to remission of grade $\geq 2$ COM to grade $<1$ was 8 days in the TJ-14 group and 15 days in the placebo group $(p=0.072)$. The hazard ratio was 1.54 [1.02 to 2.31 ] in favor of TJ-14. Treatment with TJ-14 was associated with marginally significant reduction in the duration of severe grade $\geq 2$ COM in comparison to patients receiving placebo indicating the effect of TJ-14 in reducing the severity of COM.
\end{abstract}


Conclusion: The present-pooled analysis showed that TJ-14 had a treatment effect in gastric cancer and colorectal cancer patients with COM in comparison to a placebo. Further phase III studies with a larger sample size are needed to clarify the protective effects of TJ-14 against COM.

\section{Introduction}

Gastric cancer had 951,600 new cancer cases and 723,100 deaths occurred and colorectal cancer is the third-most commonly diagnosed cancer with an estimated 1.4 million new cases and 693,900 deaths in 2012 [1]. In the treatment of gastric cancer and colorectal cancer, chemotherapy is one of the most important modalities for both the treatment of advanced cancer and the treatment of curatively resected cancer in the adjuvant setting. Although several studies have shown that chemotherapy improves and prolongs survival, it often causes severe toxicity, which affects the patient's quality of life and which may necessitate the discontinuation of chemotherapy [2-5].

Chemotherapy-induced oral mucositis (COM) is one of the most common adverse events of chemotherapy for gastric cancer and colorectal cancer [6. 7]. COM is associated with a higher risk of infection, pain, chemotherapy dose reduction, and infection-related death. The mechanisms underlying the development of COM have been investigated; one reported hypothesis is that the cyclooxygenase pathway mediates tissue injury and pain through the upregulation of pain-evoking prostaglandin E2 (PGE2) and proinflammatory cytokines [8. 9]. Despite the attempts to reduce COM, no standard efficacious prophylactic and/or therapeutic treatment has been established. Treatment is mostly supportive, consisting of good oral hygiene, mouthwashes and analgesia [10].

Hangeshashinto (TJ-14) is a traditional Japanese medicine (Kampo medicine) containing 7 herbal crude drugs. Seven herbal crude drugs are as follows: Pinelliae tuber, Scutellariae Radix, Glycyrrhizae Radix, Zizyphi Fructus, Ginseng Radix, Zingiberis Processum rhizoma, and Coptidis rhizome [11, 12]. TJ-14 has been demonstrated to directly inhibit the PGE2 production in human gingival fibroblasts and to reduce the PGE2 content in the colon in several animal models of diarrhea established using anti-cancer drugs, cholera toxin or castor oil. The use of TJ-14 resulted in the amelioration of inflammatory damage in these models [13-20]. It has also been reported that some ingredients of TJ-14 inhibit the production of PGE2 and/or the COX-2 expression.

The HANGESHA-G and HANGESHA-C trials investigated the efficacy of TJ-14 in the prevention and/or treatment of $\mathrm{COM}$ in patients receiving chemotherapy for gastric cancer and colorectal cancer, in a randomized, double-blind, placebo-controlled setting [21,22]. The current pooled analysis evaluated the efficacy of TJ-14 in the prevention and/or treatment of COM in gastric cancer and colorectal cancer using the two abovementioned prospective, multi-institutional, randomized, double-blind, placebo-controlled phase II trials.

\section{Materials and Methods}

\section{Study design}

HANGESHA-G and HANGESHA-C were prospective, multi-institutional, randomized, doubleblind, placebo-controlled phase II trials (UMIN000004214 and UMIN000004287) [21. 22]. The studies were initially designed to enroll approximately 90 patients each and to include a 4-6-week double-blind treatment period. The eligible patients were randomly assigned in a 1:1 ratio to receive either TJ-14 or the placebo. Randomization was carried out centrally at the data center using dynamic randomization with main prognostic factors, including the chemotherapy regimen (postoperative adjuvant chemotherapy, unresectable metastatic/ recurrent lesions), presence/absence of previous treatment of oral mucositis, age $(\geq 60$ years, $<60$ years) and institution. Both TJ-14 and the placebo were administered at a dose of $2.5 \mathrm{~g} /$ three times per day (for a total daily dose of $7.5 \mathrm{~g}$ ). The placebo formulation matched the texture, flavor and other characteristics of the active drug. The ingredients of placebo formulation included lactose hydrate, corn starch, dextrin, magnesium stearate, yellow No.4 aluminum lake, iron oxide red, blue No.1 aluminum lake, and caramel. The patients were advised to dissolve $2.5 \mathrm{~g}$ of TJ-14 or the placebo in $50 \mathrm{ml}$ of drinking water and rinse their oral cavity with the solution three times daily for 10 seconds. The test drug was administered from the first day to final day of the protocol treatment course. After the protocol treatment course, TJ-14 was administered for as much of one course as possible.

\section{Study population}

The detailed inclusion and exclusion criteria have been shown in the previous reports [21, 22]. Briefly, patients who were $\geq 20$ years of age and 
undergoing chemotherapy for gastric cancer or colorectal cancer were considered eligible for this study. Patients who developed moderate to severe COM (CTCAE v4.0 and WHO grade $\geq 1$ ) during any cycle of chemotherapy were asked to enroll in the study. All participants were required to have a "good" performance status (i.e., scores of 0 or 1 on the Eastern Cooperative Oncology Group performance status scale). Patients with any of the following characteristics were not eligible for the study: use of Kampo medicine within two weeks before registration (Some other Kampo medicines had mucosal protection effects); a history of severe hypersensitivity (allergy) to any medicine containing antiphlogistic, analgesics, opioids or steroids; or serious constipation (TJ-14 had some antidiarrheal effect). Pregnant or lactating women were also excluded from the study. Any other medical conditions that made a patient unsuitable for inclusion in the study according to the opinion of the investigator were also regarded as exclusion criteria (Mental disorders that might affect the ability or willingness to provide informed consent or abide by the study protocol. Systemic treatment with a corticosteroid. Systemic treatment with flucytosine, phenytoin or warfarin potassium. Pneumonitis, lung fibrosis or emphysema in need for oxygen therapy. Active inflammation due to bacteria or fungi. Unstable angina or cardiac infarction within the previous six months.)

\section{Efficacy and safety assessments}

The primary endpoint of this study was the incidence of COM (grade $\geq 2$ ) in the TJ-14 and placebo arms. The secondary endpoint was the duration of COM (grade $\geq 2$ ) in the TJ-14 and placebo arms.

\section{Statistical analyses}

All clinical data were extracted and held centrally at the Epidemiological \& Clinical Research Information Network (ECRIN) data center. The difference between the groups in the incidence of grade $\geq 2$ COM and the $90 \%$ confidence interval were calculated. Comparisons were made using the chi-squared test. The baseline characteristics were compared using the chi-squared test for categorical variables and the $t$-test test for continuous variables. The Kaplan-Meier method, log-rank test and a Cox proportional hazards regression model were used to assess the time to healing among the patients with COM. In the present study, we pre-defined the significant level as 10\%, and used 90\% CI which corresponded to significant level $10 \%$. In addition, a hazard ratio larger than 1 indicated that TJ-14 accelerated the healing of COM. The distribution of clinical factors, including age, sex, performance status, disease lesion, adjuvant or metastatic, and chemotherapy regimen were described and compared across trials using a chi-squared test or Fisher's exact test. The frequencies of adverse events were compared using Fisher's exact test. All p-values were two-sided. All statistical analyses were performed using the SAS software program (ver. 9.4 for Windows; SAS Institute, Cary, NC).

\section{Ethical considerations}

The study data and informed consent were obtained in accordance with the Declaration of Helsinki, and were approved by the Ethics Review Board of each participating institution. All patients were given a written explanation of each study protocol and provided their written informed consent before participating in the trial.

\section{Results}

\section{Patients}

The pooled population included 181 patients (HANGESHA-G [91 randomized patients; 50.3\%]; HANGESHA-C [90 patients; $49.7 \%]$ ). The baseline demographic and disease characteristics were similar across the treatment groups (Table 1). No patients received radiation therapy or molecular targeting agents before enrollment. No patients were enrolled in the study if there was any clinical evidence of another active oral mucosal disease at baseline.

Table 1. The patient characteristics of the TJ-14 and placebo groups

\begin{tabular}{|c|c|c|c|}
\hline Treatment & $\begin{array}{l}\text { TJ-14 } \\
(\mathrm{N}=88)\end{array}$ & $\begin{array}{l}\text { Placebo } \\
(\mathrm{N}=93)\end{array}$ & P-value \\
\hline Type of cancer & & & 0.822 \\
\hline Colon cancer & $43(48.9 \%)$ & $47(50.5 \%)$ & \\
\hline Gastric cancer & $45(51.1 \%)$ & $46(49.5 \%)$ & \\
\hline Sex & & & 0.296 \\
\hline Male & $55(62.5)$ & $51(54.8)$ & \\
\hline Female & $33(37.5)$ & $42(45.2)$ & \\
\hline Age & & & 0.341 \\
\hline Mean (SD) & $67.2(9.9)$ & $65.8(9.7)$ & \\
\hline Performance status & & & 0.754 \\
\hline 0 & $73(83.0)$ & $80(86.0)$ & \\
\hline 1 & 13(14.8) & 10(10.8) & \\
\hline 2 & $2(2.3)$ & $3(3.2)$ & \\
\hline Chemotherapy status & & & 0.985 \\
\hline Adjuvant setting & $33(37.5)$ & $35(37.9)$ & \\
\hline Advanced setting & $55(62.5)$ & $58(62.4)$ & \\
\hline Platinum containing chemotherapy & & & 0.494 \\
\hline Yes & $59(67 \%)$ & $66(71.7 \%)$ & \\
\hline No & $29(33 \%)$ & $26(28.3 \%)$ & \\
\hline Chemotherapy at the time of registration & & & 0.867 \\
\hline 5-fluorouracile based & $67(76.1 \%)$ & $67(72.8 \%)$ & \\
\hline Taxanes based & $3(3.4 \%)$ & $4(4.3 \%)$ & \\
\hline CPT-11 based & $18(20.5 \%)$ & $21(22.8 \%)$ & \\
\hline \multicolumn{3}{|c|}{ Dose reduction of the chemotherapy before the experimental cycles } & 0.455 \\
\hline Yes & $19(21.6 \%)$ & $16(17.2 \%)$ & \\
\hline No & $69(78.4 \%)$ & $77(82.8 \%)$ & \\
\hline
\end{tabular}




\section{The incidence of COM}

The incidence of grade $\geq 2$ COM was $55.7 \%$ (49 patients) in the TJ-14 group and $53.8 \%$ (50 patients) in the placebo group, there was no significant difference between the two groups $(\mathrm{p}=0.796)$ (Table 2). TJ-14 had no preventive effect against COM.

Table 2. The effect of TJ-14 on chemotherapy-induced oral mucositis

\begin{tabular}{llll}
\hline Treatment & $\begin{array}{l}\mathrm{TJ}-14 \\
(\mathrm{~N}=88)\end{array}$ & $\begin{array}{l}\text { Placebo } \\
(\mathrm{N}=93)\end{array}$ & P-value \\
\hline The incidence of grade $\geq 2 \mathrm{COM}^{*}$ & $49(55.7 \%)$ & $50(53.8 \%)$ & 0.796 \\
Time to remission of $\mathrm{COM}^{*}$ & $\begin{array}{l}8 \text { days } \\
\text { (range 4-10) }\end{array}$ & $\begin{array}{l}\text { 15 days } \\
\text { (range 9-24) }\end{array}$ & \\
\hline
\end{tabular}

COM: chemotherapy-induced oral mucositis

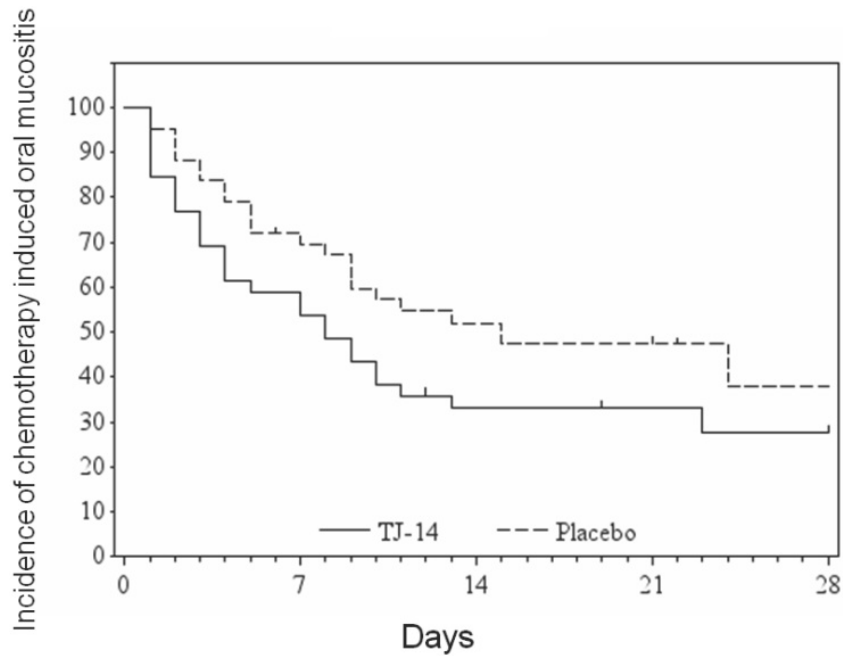

Figure 1. The duration of grade $\geq 2$ COM according to the treatment group

\section{Time to remission of COM}

The median time to remission of grade $\geq 2 \mathrm{COM}$ to grade $<1$ was 8 [ $90 \%$ confidence interval: $4-10$ ] days in the TJ-14 group and 15 [9-24] days in the placebo group $(p=0.072)$ (Figure 1) (Table 2). Treatment with TJ-14 was associated with a significant reduction in the duration of severe grade $\geq 2 \mathrm{COM}$ in comparison to the patients who received the placebo (in terms of the effect of TJ-14 in reducing the severity of COM). Moreover, the hazard ratio was 1.54 [1.02 to 2.31], in favor of TJ-14. Because a hazard ratio larger than 1 indicated that TJ-14 accelerated the healing of COM in the present study. When the subgroup analysis of time to remission of $\mathrm{COM}$, there were significant differences in patients with advanced disease and those receiving doublet regimens.

\section{Safety}

The patients' hematological parameters and blood biochemistry were analyzed and the incidence of non-hematological toxicities was investigated. The most commonly reported treatment-related adverse events were anorexia, a change in performance status, nausea, neutropenia and diarrhea, all of which typically occur in cancer patients receiving cytotoxic chemotherapy (Table 3). The majority of these events were mild to moderate in severity and considered unrelated to the study drug.

Table 3. Hematological and biochemical toxicities observed during treatment

\begin{tabular}{|c|c|c|c|c|c|c|}
\hline & \multicolumn{3}{|l|}{ Grade $\geq 1$} & \multicolumn{3}{|l|}{ Grade $\geq 2$} \\
\hline & TJ-14 & Placebo & P-value & TJ-14 & Placebo & P-value \\
\hline & $(\mathrm{N}=88)$ & $(\mathrm{N}=93)$ & & $(\mathrm{N}=88)$ & $(\mathrm{N}=93)$ & \\
\hline \multicolumn{7}{|l|}{ Hematological toxicity } \\
\hline Leucopenia & $7(8 \%)$ & $9(9.7 \%)$ & 0.796 & $0(0 \%)$ & $0(0 \%)$ & 1.000 \\
\hline Neutropenia & $8(9.1 \%)$ & $8(8.6 \%)$ & 1.000 & $1(1.1 \%)$ & $0(0 \%)$ & 0.486 \\
\hline Hemoglobin & 60 (68.2\%) & $60(64.5 \%)$ & 0.639 & $0(0 \%)$ & $0(0 \%)$ & 1.000 \\
\hline Platelet & $13(14.8 \%)$ & $16(17.2 \%)$ & 0.690 & $0(0 \%)$ & $0(0 \%)$ & 1.000 \\
\hline T-Bilirubin & $3(3.4 \%)$ & $6(6.5 \%)$ & 0.498 & $0(0 \%)$ & $0(0 \%)$ & 1.000 \\
\hline AST & $7(8 \%)$ & $7(7.5 \%)$ & 1.000 & $0(0 \%)$ & $0(0 \%)$ & 1.000 \\
\hline ALT & $9(10.2 \%)$ & $11(11.8 \%)$ & 0.815 & $0(0 \%)$ & $0(0 \%)$ & 1.000 \\
\hline \multicolumn{7}{|c|}{ Non-hematological toxicity } \\
\hline Anorexia & $38(43.2 \%)$ & $37(39.8 \%)$ & 0.654 & $3(3.4 \%)$ & $1(1.1 \%)$ & 0.357 \\
\hline Nausea & $19(21.6 \%)$ & $27(29 \%)$ & 0.306 & $2(2.3 \%)$ & $0(0 \%)$ & 0.235 \\
\hline Vomiting & $5(5.7 \%)$ & $4(4.3 \%)$ & 0.742 & $0(0 \%)$ & $0(0 \%)$ & 1.000 \\
\hline Diarrhea & $14(15.9 \%)$ & $13(14 \%)$ & 0.835 & $1(1.1 \%)$ & $1(1.1 \%)$ & 1.000 \\
\hline Constipation & $7(8 \%)$ & $13(14 \%)$ & 0.239 & $0(0 \%)$ & $0(0 \%)$ & 1.000 \\
\hline Peripheral neuropathy & $9(10.2 \%)$ & $5(5.4 \%)$ & 0.272 & $0(0 \%)$ & $1(1.1 \%)$ & 1.000 \\
\hline Lassitude & $6(6.8 \%)$ & $5(5.4 \%)$ & 0.762 & $0(0 \%)$ & $0(0 \%)$ & 1.000 \\
\hline Hand-foot syndrome & $5(5.7 \%)$ & $7(7.5 \%)$ & 0.768 & $0(0 \%)$ & $0(0 \%)$ & 1.000 \\
\hline Skin reaction & $2(2.3 \%)$ & $2(2.2 \%)$ & 1.000 & $0(0 \%)$ & $0(0 \%)$ & 1.000 \\
\hline Dysgeusia & $5(5.7 \%)$ & $1(1.1 \%)$ & 0.110 & $1(1.1 \%)$ & $0(0 \%)$ & 0.486 \\
\hline
\end{tabular}

\section{Discussion}

To date, this meta-analysis is the first evaluation of the use of TJ-14 to treat COM in patients with gastric cancer and colorectal cancer in a prospective placebo-controlled randomized study. Although the incidence of $\geq$ grade $2 \mathrm{COM}$ was similar between TJ-14 arm and the placebo arm, TJ-14 was associated with a statistically significant reduction in the duration of severe grade $\geq 2$ COM compared with patients receiving placebo in gastric cancer and colorectal cancer. Thus, TJ-14 did not have a preventive effect against COM; however, it might have had a treatment effect.

First, we want to discuss why TJ-14 did not show a preventive effect against COM in patients with gastric cancer and colorectal cancer in the present study. The most likely reason is that the dose-reduction of chemotherapy performed before the administration of TJ-14 treatment affected the incidence and duration of COM. It has been suggested that the development of mucositis is an important chemotherapy dose-limiting factor among patients with solid tumors [23]. The risk of dose reduction 
doubled when mucositis was present [24]. For example, Elting et al. retrospectively analyzed 599 patients who developed chemotherapy-induced oral mucositis [25]. They found a reduction in the dose of the next cycle of chemotherapy was twice as common after cycles with mucositis as it was after cycles without mucositis (23\% vs. $11 \%$; $\mathrm{P}<0.0001)$. Similar results were observed in another study [26]. Taking these findings into consideration, dose reduction of the chemotherapy regimen might have been a key issue in improving the incidence and/or duration of COM. We assume that the effects of TJ-14 on oral mucositis might have been offset by a reduction in the chemotherapy dose just before the experimental cycle. Actually, 20\% of the patients who developed grade $\geq 2$ COM before being entered into this study stopped or postponed the original chemotherapy and their doses were reduced at the time of the next chemotherapy cycle. Thus, these patients might have been less likely to develop COM in the present study.

Second, we wish to discuss the treatment effect of TJ-14 for COM. TJ-14 was associated with a statistically significant reduction in the duration of severe grade $\geq 2 \mathrm{COM}$ in comparison to the patients who received the placebo. TJ-14 might suppress the inflammatory cascade during COM. Previous studies showed that a statistically significant increase in the number of endothelial cells in the oral mucosa with the expression of nuclear factor kappa B (NF-kB) and cyclooxygenase 2 (COX-2) in the post-chemotherapy treatment period in comparison to that observed in the pre-treatment period. The expression of COX-2 in these cells represents the initial sign of the inflammatory cascade that determines the production of prostaglandins (PG) and further tissue damage. COX-2 is also upregulated by NF- $\mathrm{kB}$, which plays an important role in the inflammatory process [27]. TJ-14 has been demonstrated to directly inhibit the production of PGE2 and/or the expression of COX-2 [28]. Moreover, it has been previously reported that TJ-14 exerts an anti-inflammatory effect by suppressing the levels of lipopolysaccharide-induced Interleukin (IL)-6, IL-8, COX-1 and COX-2, in a dose-dependent manner [29].

The present study is associated with some limitations. First, we only focused and targeted chemotherapy-induced oral mucositis (especially cytotoxic agent-induced oral mucositis) in the present study. Molecular targeting therapy and radiotherapy are well-established treatments for gastric cancer and colorectal cancer. However, although these treatments also induce mucositis, patients who received these treatments were not included in the present study. Thus, it is unclear whether the present study results are applicable to molecular targeting treatment- induced oral mucositis and/or radiation-induced oral mucositis. Second, the incidence of COM might have been underestimated. In the present study, the physicians checked the oral condition and investigated the incidence of COM in all patients at in an outpatients setting during the pre-treatment and experimental periods according to the WHO or CTCAE criteria. However, the physicians were able to detect only severe pseudo membranous/ulcerative mucositis and could not detect mild to moderate COM, which is often misdiagnosed as pain or numbness. Third, cryotherapy is widely recommended as a promising method for COM. However, we did not compare the effect of TJ-14 and placebo with cryotherapy. Further study should be focus on this issue.

In conclusion, although this trial did not show a beneficial for prevent effect of TJ-14 in reducing COM, the treatment effect of TJ-14 was associated with a statistically significant reduction in the duration of severe grade $\geq 2 \mathrm{COM}$ in comparison to patients who received a placebo. TJ-14 might have a treatment effect in COM. Further study should focus on the treatment effect of TJ-14 in COM.

\section{Acknowledgments}

This study was supported in part by a non-profit organization, the Epidemiological \& Clinical Research Information Network (ECRIN).

\section{Conflict of Interest Statement}

The authors declare no conflicts of interest in association with the present study.

\section{References}

1. Torre LA, Bray F, Siegel RL, Ferlay J, Lortet-Tieulent J, Jemal A. Global cancer statistics, 2012. CA Cancer J Clin. 2015; 65: 87-108.

2. Cunningham D, Allum WH, Stenning SP, Thompson JN, Van de Velde CI, Nicolson $\mathrm{M}$, et al. Perioperative chemotherapy versus surgery alone for resectable gastro esophageal cancer. N Engl J Med 2006; 355: 11-20.

3. Sakuramoto S, Sasako M, Yamaguchi T, Kinoshita T, Fujii M, Nashimoto A, et al. Adjuvant chemotherapy for gastric cancer with S-1, an oral fluoropyrimidine. N Engl J Med 2007; 357: 1810-1820.

4. Yoshida M, Ishiguro M, Ikejiri K, Mochizuki I, Nakamoto Y, Kinugasa Y, et al. S-1 as adjuvant chemotherapy for stage III colon cancer: a randomized phase III study (ACTS-CC trial). Ann Oncol. 2014; 25: 1743-9.

5. de Gramont A, Figer A, Seymour M, Homerin M, Hmissi A, Cassidy J, et al. Leucovorin and fluorouracil with or without oxaliplatin as first-line treatment in advanced colorectal cancer. J Clin Oncol. 2000; 18: 2938-47.

6. Keefe DM, Schubert MM, Elting LS, Sonis ST, Epstein JB, Raber-Durlacher JE, et al. Mucositis Study Section of the Multinational Association of Supportive Care in Cancer and the International Society for Oral Oncology. Updated clinical practice guidelines for the prevention and treatment of mucositis. Cancer. 2007; 109: 820-31.

7. Elting LS, Keefe DM, Sonis ST, Garden AS, Spijkervet FK, Barasch A, et al. Burden of Illness Head and Neck Writing Committee. Patient-reported measurements of oral mucositis in head and neck cancer patients treated with radiotherapy with or without chemotherapy: demonstration of increased frequency, severity, resistance to palliation, and impact on quality of life. Cancer. 2008; 113: 2704-13.

8. Lalla RV(1), Pilbeam CC, Walsh SJ, Sonis ST, Keefe DM, Peterson DE. Role of the cyclooxygenase pathway in chemotherapy-induced oral mucositis: a pilot study. Support Care Cancer. 2010; 18: 95-103.

9. Morales-Rojas T, Viera N, Morón-Medina A, Alvarez CJ, Alvarez A. Proinflammatory cytokines during the initial phase of oral mucositis in 
patients with acute lymphoblastic leukaemia. Int J Paediatr Dent. 2012; 22: 191-6

10. Rubenstein EB, Peterson DE, Schubert M, Keefe D, McGuire D, Epstein J, et al. Mucositis Study Section of the Multinational Association for Supportive Care in Cancer; International Society for Oral Oncology. Clinical practice guidelines for the prevention and treatment of cancer therapy-induced oral and gastrointestinal mucositis. Cancer. 2004; 100: 2026-46.

11. Kono T, Kanematsu T, Kitajima M. Exodus of Kampo, traditional Japanese medicine, from the complementary and alternative medicines: is it time yet? Surgery 2009; 146: 837-840.

12. Mori K1, Kondo T, Kamiyama Y, Kano Y, Tominaga K. Preventive effect of Kampo medicine (Hangeshashin-to) against irinotecan-induced diarrhea in advanced non-small-cell lung cancer. Cancer Chemother Pharmacol 2003; 51: 403-406

13. Nakazono Y, Ara T, Fujinami Y, Hattori T, Wang PL: Preventive Effects of a Kampo Medicine, Hangeshashinto on Inflammatory Responses in Lipopolysaccharide-Treated Human Gingival Fibroblasts. J Hard Tissue Biol 2010, 19(1):43-50.

14. Kase Y, Saitoh K, Yuzurihara M, Ishige A, Komatsu Y: Effects of Hange-shashin-to on cholera toxin-induced fluid secretion in the small intestine of rats. Biol Pharm Bull 1998, 21(2):117-120.

15. Kase Y, Saitoh K, Makino B, Hashimoto K, Ishige A, Komatsu Y: Relationship between the antidiarrhoeal effects of Hange-Shashin-To and its active components. Phytother Res 1999, 13(6):468-473.

16. Lantz RC, Chen GJ, Sarihan M, Solyom AM, Jolad SD, Timmermann BN: The effect of extracts from ginger rhizome on inflammatory mediator production. Phytomedicine 2007, 14(2-3):123-128.

17. Pan MH, Hsieh MC, Hsu PC, Ho SY, Lai CS, Wu H, Sang S, Ho CT: 6-Shogaol suppressed lipopolysaccharide-induced up-expression of iNOS and COX-2 in murine macrophages. Mol Nutr Food Res 2008, 52(12):1467-1477.

18. Nievergelt A Marazzi J, Schoop $\mathrm{R}$, Altmann $\mathrm{KH}$, Gertsch J: Ginger phenylpropanoids inhibit IL-1beta and prostanoid secretion and disrupt arachidonate-phospholipid remodeling by targeting phospholipases A2. J Immunol 2011, 187(8):4140-4150.

19. Jeong HW, Hsu KC, Lee JW, Ham M, Huh JY, Shin HJ, Kim WS, Kim JB: Berberine suppresses proinflammatory responses through AMPK activation in macrophages. Am J Physiol Endocrinol Metab 2009, 296(4E): 955-964.

20. Feng AW, Gao W, Zhou GR, et al. Berberine ameliorates COX-2 expression in rat small intestinal mucosa partially through PPARgamma pathway during acute endotoxemia. Int Immunopharmacol. 2012; 12(1): 182-188.

21. Aoyama T, Nishikawa K, Takiguchi N, Tanabe K, Imano M, Fukushima R, et al. Double-blind, placebo-controlled, randomized phase II study of TJ-14 (hangeshashinto) for gastric cancer chemotherapy-induced oral mucositis. Cancer Chemother Pharmacol. 2014; 73: 1047-54.

22. Matsuda C, Munemoto Y, Mishima H, Nagata N, Oshiro M, Kataoka M, et al. Double-blind, placebo-controlled, randomized phase II study of TJ-14 (Hangeshashinto) for infusional fluorinated-pyrimidine-based colorectal cancer chemotherapy-induced oral mucositis. Cancer Chemother Pharmacol. 2015; 76: 97-103.

23. Sonis ST, Elting LS, Keefe D, Peterson DE, Schubert M, Hauer-Jensen M, et al. Perspectives on cancer therapy-induced mucosal injury: pathogenesis, measurement, epidemiology, and consequences for patients. Cancer. 2004; 100: 1995-2025.

24. Keefe DM, Schubert MM, Elting LS, Sonis ST, Epstein JB, Raber-Durlacher JE, et al. Updated clinical practice guidelines for the prevention and treatment of mucositis. Cancer. 2007; 109: 820-31.

25. Elting LS, Cooksley C, Chambers M, Cantor SB, Manzullo E, Rubenstein EB. The burdens of cancer therapy. Clinical and economic outcomes of chemotherapy-induced mucositis. Cancer. 2003; 98: 1531-9.

26. Coleman RE, Biganzoli L, Canney P, Dirix L, Mauriac L, Chollet P, et al. A randomised phase II study of two different schedules of pegylated liposomal doxorubicin in metastatic breast cancer (EORTC-10993). Eur J Cancer. 2006; 42: 882-7.

27. Kase Y, Hayakawa T, Ishige A, Aburada M, Komatsu Y. The effects of Hange-shashin-to on the content of prostaglandin E2 and water absorption in the large intestine of rats. Biol Pharm Bull. 1997; 20: 954-7.

28. Kase Y, Saitoh K, Ishige A, Komatsu Y. Mechanisms by which Hange-shashin-to reduces prostaglandin E2 levels. Biol Pharm Bull. 1998; 21: 1277-81.

29. Nakazono Y, Ara T, Fujinami Y, Hattori T, Wang P L. Preventive effects of a Kampo, Hangeshashinto on inflammatory responses in lipopolysaccharide-treated human gingival fibroblasts. J Hard Tissue Biology. 2010; 19: 43-50.

30. Aoyama T, Nishikawa K, Oba M, et al. Meta-analysis of Patient-level Data on Therapeutic Effects of TJ-14 (Hangeshashinto) for Gastroenterological Cancer Chemotherapy-induced Severe Oral Mucositis with the HANGESHA-G and HANGESHA-Cs: protocol paper. Ann. Cancer Res. Ther. 2017; 25( No. 2): 92-94. 